\title{
BCS-BEC crossover in a relativistic superfluid and its significance to quark matter
}

\author{
Yusuke Nishida, , and Hiroaki Abuki ${ }^{2}$, \\ ${ }^{1}$ Department of Physics, University of Tokyo, Tokyo 113-0033, Japan \\ ${ }^{2}$ Yukawa Institute for Theoretical Physics, Kyoto University, Kyoto 606-8502, Japan
}

(Dated: September 18, 2018)

\begin{abstract}
The character change of a superfluid state due to the variation of the attractive force is investigated in the relativistic framework with a massive fermion. Two crossovers are found. One is a crossover from the usual BCS state to the Bose-Einstein condensation (BEC) of bound fermion pairs. The other is from the BEC to the relativistic Bose-Einstein condensation (RBEC) of nearly massless bound pairs where antiparticles as well as particles dominate the thermodynamics. Possible realization of the BEC and RBEC states in the quark matter is also pointed out.
\end{abstract}

PACS numbers: 74.20.Fg, 03.75.Nt, 11.10.Wx, 12.38.-t

Recently, new superfluid states in the ultracold gas of fermionic alkali atoms $\left({ }^{40} \mathrm{~K},{ }^{6} \mathrm{Li}\right)$ were realized [1]. Using the Feshbach resonance, the long-standing idea of the crossover from the BCS state to the Bose-Einstein condensation (BEC) 2, 3, 4] has been extensively examined. The basic concept of the BCS-BEC crossover is as follows: As long as the attractive interaction between fermions is weak, the system exhibits the superfluidity characterized by the energy gap in the BCS mechanism. On the other hand, if the attractive interaction is strong enough, the fermions first form bound molecules (bosons). Then they start to condense into the bosonic zero-mode at some critical temperature. These two situations are smoothly connected without the phase transition.

The possible realization of the BCS-BEC crossover in various systems has been theoretically investigated. These include the liquid ${ }^{3} \mathrm{He}[3]$, the trapped alkali atoms [5], and the nuclear matter [6]. One of the most striking features of the crossover is that the critical temperature in the BEC region is independent of the coupling for the attraction between fermions. This is because the increase of the coupling only affects the internal structure of the bosons, while the critical temperature is determined by the boson's kinetic energy. Thus, the critical temperature reaches a ceiling for the large coupling as long as the binding effect on the boson mass can be neglected. Even in the nuclear matter where the interaction is relatively strong, the binding energy of the deuteron is much smaller than the nucleon mass. This fact allows us to work within a nonrelativistic framework for describing such a crossover.

It is interesting to ask how the situation changes in relativistic systems where the binding effect can not be neglected. The color superconducting phase in the dense quark matter $[7,8]$ and the pion superfluid phase at finite isospin density [9] would be examples. In this article, we will show that there could be two crossovers in the relativistic superfluids. One is the ordinary BCS-BEC

\footnotetext{
${ }^{*}$ E-mail: nishida@nt.phys.s.u-tokyo.ac.jp

${ }^{\dagger}$ E-mail: abuki@yukawa.kyoto-u.ac.jp
}

crossover, where the critical temperature in the BEC region would not plateau because of the relativistic effect. The other is from the BEC state to the novel state, the relativistic $B E C$ ( $R B E C)$, where the critical temperature increases to the order of the Fermi energy.

In order to explore the BCS-BEC and BEC-RBEC crossovers in the relativistic system, we start with the following contact four-Fermi interaction model:

$$
\begin{aligned}
\mathcal{L}[\psi, \bar{\psi}]=\bar{\psi} & \left(i \not \partial-m+\gamma_{0} \mu\right) \psi \\
& +G\left(i \psi^{\dagger} \gamma_{5} C \psi^{*}\right) \cdot\left(i \psi^{\mathrm{T}} \gamma_{5} C \psi\right) .
\end{aligned}
$$

Here $\psi=\psi(t, \boldsymbol{x})$ is a Dirac fermion field having a spinor index implicitly. The fermion mass and chemical potential are denoted by $m$ and $\mu . C=i \gamma_{0} \gamma_{2}$ is the charge conjugation matrix and $G$ is a coupling constant for the attraction in the $J^{P}=0^{+}$channel. Qualitative results shown below are not modified even when fermions have internal degrees of freedom other than spin. Therefore, we will make our analysis without them for simplicity.

The partition function can be written as

$$
Z=\int \mathcal{D} \psi \mathcal{D} \bar{\psi} \exp \left(-\int_{0}^{1 / T} d \tau \int d \boldsymbol{x} \mathcal{L}_{\mathrm{E}}[\psi, \bar{\psi}]\right),
$$

where $\mathcal{L}_{\mathrm{E}}$ is the Lagrangian density in the Euclidean space. Introducing Hubbard-Stratonovich fields $\Delta(\tau, \boldsymbol{x})$ for $i \psi^{\mathrm{T}} \gamma_{5} C \psi$ and $\Delta^{*}(\tau, \boldsymbol{x})$ for $i \psi^{\dagger} \gamma_{5} C \psi^{*}$ and integrating out the fermion fields lead to

$$
Z=Z_{0} \int \mathcal{D} \Delta \mathcal{D} \Delta^{*} \exp \left(-S_{\text {eff }}\left[\Delta, \Delta^{*}\right]\right)
$$

Here $Z_{0}=e^{-\beta \Omega_{0}(\mu, T)}$ is the free fermion part of the partition function, while $S_{\text {eff }}\left[\Delta, \Delta^{*}\right]$ is the effective action for the bosonic fields. In order to include the effect of the fluctuation, we evaluate the functional integral in the Gaussian approximation, whose validity will be discussed later. Expansion of the effective action up to the second order in $\Delta$ results in

$$
S_{\mathrm{eff}}\left[\Delta, \Delta^{*}\right] \simeq T \sum_{n} \int \frac{d \boldsymbol{p}}{(2 \pi)^{3}}\left[\frac{1}{G}-\chi\left(i \omega_{n}, \boldsymbol{p}\right)\right]\left|\tilde{\Delta}\left(i \omega_{n}, \boldsymbol{p}\right)\right|^{2},
$$


where $\tilde{\Delta}\left(i \omega_{n}, \boldsymbol{p}\right)$ is the momentum representation of the pair field with $\omega_{n}=2 \pi n T$ being the Matsubara frequency. $\chi\left(i \omega_{n}, \boldsymbol{p}\right)$ is a pair susceptibility at the one loop level [10]. The critical temperature for the superfluidity, $T_{\mathrm{c}}$, is given by the solution of the equation:

$$
\frac{1}{G}-\left.\chi(0, \mathbf{0})\right|_{T=T_{\mathrm{c}}}=0 .
$$

This is nothing but the Thouless criterion which states that the pair fluctuation becomes tachyonic at low momentum because of $1 / G-\left.\chi(0, \mathbf{0})\right|_{T<T_{\mathrm{c}}}<0$. This is the signal of the BCS instability to the formation of Cooper pairs.

The integration over $\Delta, \Delta^{*}$ in Eq. (3) leads to the thermodynamic potential in the Gaussian approximation: $\Omega(\mu, T)=\Omega_{0}(\mu, T)+\Omega_{\text {fluc }}(\mu, T)$ with

$$
\Omega_{\mathrm{fluc}}(\mu, T)=T \sum_{n} \int \frac{d \boldsymbol{p}}{(2 \pi)^{3}} \log \left[\frac{1}{G}-\chi\left(i \omega_{n}, \boldsymbol{p}\right)\right] .
$$

Following Nozières and Schmitt-Rink [4], $\Omega_{\text {fluc }}$ can be written in terms of a phase shift $\delta(\omega, \boldsymbol{p})$ defined by $\delta(\omega, \boldsymbol{p})=-\arg [1 / G-\chi(\omega+i 0, \boldsymbol{p})]$. By differentiating the thermodynamic potential with $\mu$, we obtain the fermion number density as follows:

$$
\begin{aligned}
N_{\text {total }}= & 2 \int \frac{d \boldsymbol{p}}{(2 \pi)^{3}}\left\{f_{\mathrm{F}}\left(E_{\boldsymbol{p}}-\mu\right)-f_{\mathrm{F}}\left(E_{\boldsymbol{p}}+\mu\right)\right\} \\
& +\int \frac{d \boldsymbol{p}}{(2 \pi)^{3}} \int_{-\infty}^{\infty} \frac{d \omega}{\pi} f_{\mathrm{B}}(\omega) \frac{\partial \delta}{\partial \mu}(\omega, \boldsymbol{p})
\end{aligned}
$$

with $f_{\mathrm{F}}(\omega)=1 /(\exp [\omega / T]+1)$ being the Fermi distribution function and $f_{\mathrm{B}}(\omega)=\operatorname{sign}(\omega) /(\exp [|\omega| / T]-1)$ being the Bose distribution function. ${ }^{1}$ The first term which we denote by $N_{\mathrm{MF}}=N_{\mathrm{F}}-N_{\overline{\mathrm{F}}}$ represents the contribution of fermions and antifermions at the mean field level and the second one which we denote by $N_{\text {fluc }}$ represents the contribution of pair fluctuations. Instead of $N_{\text {total }}$, we will sometimes use the Fermi momentum $p_{\mathrm{F}}$, which is defined by $N_{\text {total }}=p_{\mathrm{F}}^{3} / 3 \pi^{2}$.

If the attraction is strong enough, bound states appear and we can extract the bound boson (antiboson) contribution $N_{\mathrm{B}}\left(N_{\overline{\mathrm{B}}}\right)$ from $N_{\text {fluc }}[4,[5]$. By picking up the bound state poles in $\partial \delta(\omega, \boldsymbol{p}) / \partial \mu$ in the $\omega$-integral of Eq. (7), we obtain

$$
N_{\mathrm{B}}=\int \frac{d \boldsymbol{p}}{(2 \pi)^{3}}\left[2-\frac{\partial \omega_{\mathrm{B}}(\boldsymbol{p})}{\partial \mu}\right] f_{\mathrm{B}}\left(\omega_{\mathrm{B}}(\boldsymbol{p})-2 \mu\right)
$$

and

$$
N_{\overline{\mathrm{B}}}=\int \frac{d \boldsymbol{p}}{(2 \pi)^{3}}\left[2+\frac{\partial \omega_{\overline{\mathrm{B}}}(\boldsymbol{p})}{\partial \mu}\right] f_{\mathrm{B}}\left(\omega_{\overline{\mathrm{B}}}(\boldsymbol{p})+2 \mu\right) .
$$

\footnotetext{
${ }^{1}$ We use this Bose distribution function so that the thermodynamic potential is symmetric under $\mu \rightarrow-\mu$.
}

Here, $\omega_{\mathrm{B}}(\boldsymbol{p})$ and $-\omega_{\overline{\mathrm{B}}}(\boldsymbol{p})$ are the solutions of $1 / G-\chi(\omega-$ $2 \mu, \boldsymbol{p})=0$ and correspond to the energy of the boson and the antiboson, respectively. Then the remaining part $N^{\text {unstable }}=N_{\text {fluc }}-\left(N_{\mathrm{B}}-N_{\overline{\mathrm{B}}}\right)$ can be interpreted as the contribution of unstable off-shell bosons.

In numerical calculations, a momentum cutoff $\Lambda$ is introduced in order to regularize the ultraviolet divergence and all the dimensionful quantities are scaled by $\Lambda$. We take a characteristic parameter set $(m / \Lambda=0.2$ and $p_{\mathrm{F}} / \Lambda=0.1$ ) so that we can analyze the effect of relativity. We confirmed that the variation of $p_{\mathrm{F}}$ does not change our qualitative arguments below. Also, how the variation of $m$ affects our results will be discussed later. Figures \(a) and प(b) show numerical results of the critical temperature $T_{\mathrm{c}}$ and the chemical potential $\mu$ as functions of $G$ with the total number density $N_{\text {total }}$ fixed, which are obtained by solving Eqs. (5) and (7) simultaneously. The ratios of the fermion and stable boson densities to $N_{\text {total }}$ at $T=T_{\mathrm{c}}$ are also plotted in Fig. 1(c). Based on these three figures, we will argue that there are three physically distinct regions; the weak, intermediate, and strong coupling regions. The superfluid states realized in the three regions will be interpreted as the BCS, $\mathrm{BEC}$, and relativistic BEC phases, respectively.

In the weak coupling region $G / G_{0} \lesssim 0.86, T_{\mathrm{c}}$ increases exponentially as is well-known in the weak coupling BCS theory. Its behavior is well described by the mean field approximation (the left thin solid line in Fig. 1(a)),$\mu$ in this region is almost equal to the Fermi energy $E_{\mathrm{F}}=\sqrt{m^{2}+p_{\mathrm{F}}^{2}}$. Accordingly, the fermion density $N_{\mathrm{F}}$ dominates the total density. From these facts, the superfluid state realized in this region can be regarded as the BCS state.

In the intermediate coupling region $0.86 \lesssim G / G_{0} \lesssim$ 1.07, $T_{\mathrm{c}}$ increases much slowly and $\mu$ decreases monotonously. Once $\mu$ becomes smaller than $m$, stable bosons with the mass $M_{\mathrm{B}}\left(T_{\mathrm{c}}\right)=2 \mu$ appear and they dominate the total density. ${ }^{2}$ The critical temperature for the ideal Bose gas is approximately given by

$$
T_{\mathrm{BEC}}^{\mathrm{NR}}=\frac{2 \pi}{M_{\mathrm{B}}}\left[\frac{N_{\mathrm{B}}}{2 \zeta(3 / 2)}\right]^{2 / 3} .
$$

This nonrelativistic formula for the BEC critical temperature with the boson mass $M_{\mathrm{B}}\left(T_{\mathrm{c}}\right)=2 \mu$ is examined by the dotted line in Fig. 1(a), which well approximates $T_{\mathrm{c}}$ in the intermediate coupling region. Therefore, we can interpret the superfluid state realized in this region is in the BEC phase. The slowly increasing $T_{\mathrm{c}}$ in the BEC phase is in contrast to the result of the nonrelativistic calculation where the critical temperature approaches to a constant value 11]. In the nonrelativistic framework, the change of the boson mass due to the binding can be

\footnotetext{
2 The apparent singularity in the stable boson density in Fig. 11 (c) does not mean a phase transition. The total boson density $N_{\text {fluc }}$ is a smooth and positive function of the coupling.
} 
neglected by definition. Thus, the critical temperature in the BEC phase is given by $T_{\mathrm{BEC}}^{\mathrm{NR}}$ with $M_{\mathrm{B}}=2 m$, which is indicated by the arrow in Fig. 1(a), and is independent of the coupling. In our relativistic framework, however, the boson mass $M_{\mathrm{B}}\left(T_{\mathrm{c}}\right)=2 \mu$ can become smaller, and consequently, $T_{\mathrm{c}}$ becomes larger as one increases the coupling.

We remark that the criterion for the BEC state, $\mu<$ $m$, is model independent. At $T=T_{\mathrm{c}}$ of the BEC, the chemical potential for the boson $\mu_{\mathrm{B}}=2 \mu$ should be equal to its mass $M_{\mathrm{B}}$. On the other hand, $M_{\mathrm{B}}$ must be less than $2 m$ for the binding. Therefore, we have $\mu<m$ in the BEC region.

Let us discuss the strong coupling region $G / G_{0} \gtrsim 1.07$, where $T_{\mathrm{c}}$ rapidly increases and the nonrelativistic formula for the BEC critical temperature breaks down. Because $\mu$ is smaller than $T_{\mathrm{c}}$ in this region, antiparticles can be easily excited. As is shown in Fig. प(c), the antifermion and antiboson densities $\left(N_{\overline{\mathrm{F}}}, N_{\overline{\mathrm{B}}}\right)$ grow rapidly. At the same time, the fermion and stable boson densities $\left(N_{\mathrm{F}}, N_{\mathrm{B}}\right)$ increase so that the total number density is unchanged. $T_{\mathrm{c}}$ in this region can be approximated by the ideal BEC critical temperature in the relativistic limit [12]:

$$
T_{\mathrm{BEC}}^{\mathrm{RL}}=\sqrt{\frac{3\left(N_{\mathrm{B}}-N_{\overline{\mathrm{B}}}\right)}{2 M_{\mathrm{B}}}} .
$$

We note this approximate formula slightly deviates from $T_{\mathrm{c}}$ particularly in the large coupling region. This is because a large number of fermions are accompanied there, which is favorable in terms of entropy. We refer to the boson condensed phase with antiparticles in the strong coupling region as the relativistic BEC (RBEC) phase.

We can also understand the rapid increase of $T_{\mathrm{c}}$ in terms of the decreasing mean interparticle distance $\bar{d}_{\mathrm{p}}$. Let us estimate the critical temperature in the RBEC phase by comparing the thermal de Broglie wavelength $\pi /(\sqrt{3} T)$ to $\bar{d}_{\mathrm{p}}$. We estimate $\bar{d}_{\mathrm{p}}$ by $N_{\mathrm{F}}^{-1 / 3}$ since fermions give a dominant contribution to the density in the strong coupling region. Thus, we have $T \sim \pi N_{\mathrm{F}}^{1 / 3} / \sqrt{3}$, which agrees with $T_{\mathrm{c}}$ well (see the right thin solid line in Fig. 1(a)).

An essential difference between the BCS and (R)BEC phases is that the stable bosons are present above $T_{\mathrm{c}}$ in the (R)BEC region. As the temperature is increased, the binding energy of the stable boson decreases. The bound state pole eventually disappears at a certain temperature, which we call a dissociation temperature $T_{\text {diss }}$. $T_{\text {diss }}$ as a function of the coupling is shown in Fig. 1(a) by the dashed line. $T_{\text {diss }}$ line appears from the point $G / G_{0} \simeq 0.92$ where the fermion pairs start to form the bound bosons. They get bound deeper with the increasing coupling, and as a consequence, $T_{\text {diss }}$ increases monotonically. $T_{\text {diss }}$ line separates the normal phase into two regions; a normal phase without stable bosons for $T>T_{\text {diss }}$, and a preformed boson phase with stable bosons for $T_{\mathrm{c}}<T<T_{\text {diss. }}$. The preformed boson phase in the intermediate and strong coupling regions may pro-

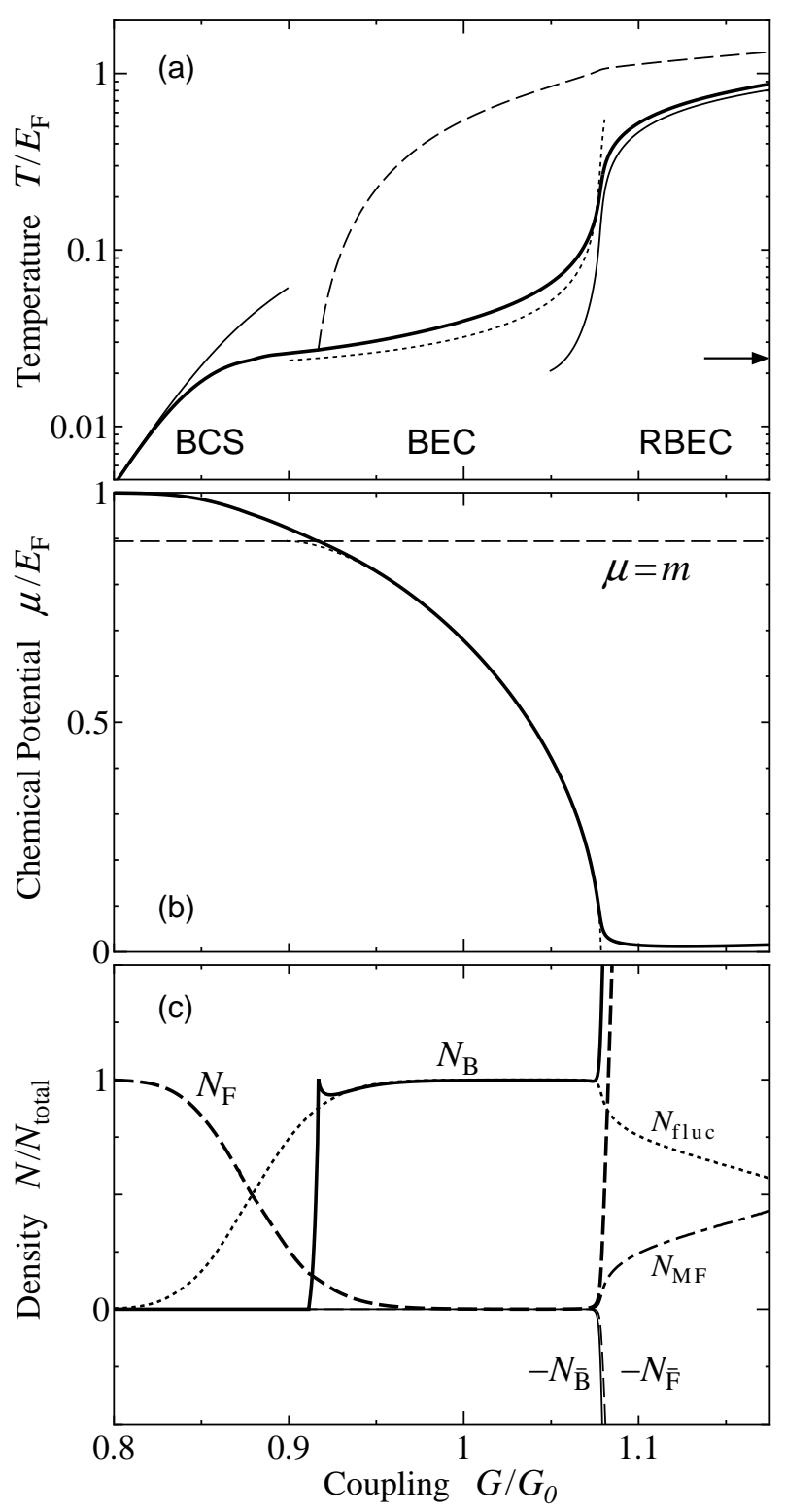

FIG. 1: (a) Critical temperature $T_{\mathrm{c}}$ normalized by the Fermi energy $E_{\mathrm{F}}=\sqrt{m^{2}+p_{\mathrm{F}}^{2}}$ (thick solid line) as a function of the coupling $G / G_{0}$. $G_{0}$ is defined by $G_{0}=\pi^{2} / \Lambda^{2}$. For other lines, see the text. (b) Chemical potential $\mu / E_{\mathrm{F}}$ as a function of the coupling $G / G_{0}$. The dashed line represents the level where $\mu=m ; m / E_{\mathrm{F}} \simeq 0.89$ in the present case. The dotted line corresponds to one half of the bound boson's mass in the vacuum. (c) The ratios of $N_{\mathrm{F}}, N_{\overline{\mathrm{F}}}, N_{\mathrm{B}}$ and $N_{\overline{\mathrm{B}}}$ to the fixed $N_{\text {total }}$ as functions of $G / G_{0} . N_{\mathrm{MF}} / N_{\text {total }}$ and $N_{\text {fluc }} / N_{\text {total }}$ are also plotted. The line for $N_{\mathrm{MF}}$ is behind that for $N_{\mathrm{F}}$ for $G / G_{0} \lesssim 1.07$ because of the absence of antifermions.

vide us with new insight into the precursory phenomenon above $T_{\mathrm{c}}$ 10.

We have discussed the character change of the superfluid state with a specific set of $m$ and $p_{\mathrm{F}}$. Let us now discuss the $m$-dependence of the crossover boundaries with keeping $p_{\mathrm{F}}$ fixed. The crossover boundary from the BCS 
to the $\mathrm{BEC}$ is characterized by the point where the bound states are formed in the medium. On the other hand, the crossover boundary from the BEC to the RBEC is characterized by the point where the boson mass $2 \mu$ becomes smaller than $T_{\mathrm{c}}$. These two points are well approximated by the coupling $G_{1}$ where the boson is formed and $G_{2}$ where it becomes massless in the vacuum with $\mu=T=0$ (see the dotted line in Fig. 1(b) which is indistinguishable from the solid line). We can show that $G_{1(2)}$ increases (decreases) as decreasing the fermion mass $m$. It means that it becomes hard to bind two fermions due to the larger kinetic energy for a smaller $m$, while less attraction will be needed to cancel $2 m$ by the binding. Accordingly, the BEC region shrinks with decreasing $m$, while the RBEC dominates the larger region in the coupling space. In the ultrarelativistic limit $m \rightarrow 0$, the BEC region will disappear because fermions could no longer be bound. Even in this case, we still have a superfluid phase with $2 \mu<T_{\text {c }}$ for the large coupling which is smoothly connected with the RBEC phase at $m>0$.

In summary, we have discussed two crossovers in the relativistic four-Fermi model with the massive fermion: One is the crossover from the usual BCS to the BEC of bound fermion pairs and the other is that from the $\mathrm{BEC}$ to the RBEC of nearly massless bound pairs. In order to avoid the cutoff artifacts, we have checked each of $N_{\mathrm{F}}, N_{\mathrm{B}}, N_{\overline{\mathrm{F}}}$ and $N_{\overline{\mathrm{B}}}$ does not exceed $(\Lambda / 2 \pi)^{3}$ within the coupling range shown here.

Since we have employed the Gaussian approximation which corresponds to the resummation of ring diagrams into the thermodynamic potential, all the interactions among bosons are neglected in our analysis. However, it is shown in the nonrelativistic framework that the 2body interaction between bosons becomes smaller with increasing the attraction between fermions [11]. The multibody scatterings among bosons are also negligible in a dilute gas. Thus, our approximation is valid except for the vicinity of the BCS-BEC crossover boundary and the very dense RBEC limit. Going beyond the Gaussian approximation so as to take into account the interactions among bosons in our relativistic framework is an important future issue. Other approaches to the BCS-BEC crossover also may be useful [13].

Finally, we make some speculative remarks on the relevance of the phases discussed above to QCD. The BCSBEC crossover, which takes place for $\mu \gg T$, may be realized in the cold dense quark matter [8]. The fermion mass $m$ in Eq. (1) in this case should be interpreted as the current or dynamical quark masses. Also, it is an interesting future problem to generalize our model by taking into account the plasmino mass $m_{\mathrm{pl}} \sim g \sqrt{\mu^{2}+\pi^{2} T^{2}}$ with $g$ being the QCD coupling constant. The plasmino mass can play a role of the chiral invariant mass constituting the boson mass. Further study with the plasmino effective action [14] will give us more insight into the realistic BCS-BEC crossover in the quark matter. In fact, the BEC criterion $\mu<m_{\text {pl }}$ leads to $g \gtrsim 1$ for $\mu \gg T$, which corresponds to the density relevant to the center of compact stars.

The BEC-RBEC crossover, which takes place for $T \gg$ $\mu \sim 0$, will be relevant to the quark-gluon plasma just above the deconfinement transition. Possibility of having not only $q \bar{q}$ bound states 15 but also $q q$ bound states 16] in the deconfined phase has close relevance to the bound bosons in the RBEC state in this article. The plasmino mass $m_{\mathrm{pl}} \sim g T$ again will play a crucial role to have bound bosons in the realistic situation.

The above discussions suggest that there is a band of superfluid phases (BCS-BEC-RBEC) between the hadronic phase and the quark-gluon plasma phase in the QCD phase diagram. Also, the preformed boson phase may exist between the $(\mathrm{R}) \mathrm{BEC}$ phase and the quarkgluon plasma phase. The transport properties in these phases are also of great interest. In the nonrelativistic framework, it is shown that the pair fluctuation is dissipative in the BCS region, while it propagates without viscous damping in the BEC region [11]. The detailed analysis including all the relevant hydrodynamic modes may provide a picture for almost the perfect fluid aspect of quark-gluon plasma.

The generalization of our work so as to allow for the $q \bar{q}$ condensation will be essential to see the realization of the BEC and RBEC states in QCD. It is known that the one-gluon exchange generates the attraction in the scalar $q \bar{q}$ channel whose strength is 2 times larger than that in the $q q$ channel. Even though the $q q$ condensation still has a kinematic advantage due to the existence of the Fermi surface, the dominant $q \bar{q}$ attraction may wash out the (R)BEC phase leading to the large $q \bar{q}$ condensation. Whether the (R)BEC phase survives in the QCD phase diagram should be settled after taking into account the possibility of the $q \bar{q}$ condensation in our analysis.

\section{Acknowledgments}

The authors would like to thank T. Hatsuda for discussions, comments and reading manuscript. Y. N. is supported by the Japan Society for the Promotion of Science for Young Scientists. H. A. is supported by the 21COE program "Center for Diversity and Universality in Physics" at Kyoto University.
[1] C.A. Regal, M. Greiner, and D.S. Jin, Phys. Rev. Lett. 92, 040403 (2004); M. Bartenstein et al., Phys. Rev. Lett.
92, 120401 (2004); M.W. Zwierlein et al., Phys. Rev. Lett. 92, 120403 (2004). 
[2] D.M. Eagles, Phys. Rev. 186, 456 (1969).

[3] A.J. Leggett, J. Phys. C 41, 7 (1980).

[4] P. Nozières and S. Schmitt-Rink, J. Low Temp. Phys. 59, 195 (1985).

[5] Y. Ohashi and A. Griffin, Phys. Rev. Lett. 89, 130402 (2002); Phys. Rev. A 67, 033603 (2003).

[6] H. Stein et al., Z. Phys. A 351, 295 (1995).

[7] For reviews, see K. Rajagopal and F. Wilczek, arXiv:hep-ph/0011333 M.G. Alford, Annu. Rev. Nucl. Part. Sci. 51, 131 (2001).

[8] H. Abuki, T. Hatsuda, and K. Itakura, Phys. Rev. D 65, 074014 (2002); K. Itakura, Nucl. Phys. A 715, 859 (2003).

[9] D.T. Son and M.A. Stephanov, Phys. Rev. Lett. 86, 592 (2001); Phys. At. Nucl. 64, 834 (2001).

[10] M. Kitazawa et al., Phys. Rev. D 65, 091504(R) (2002); Phys. Rev. D 70, 056003 (2004).

[11] C.A.R. Sá de Melo, M. Randeria, and J.R. Engelbrecht,
Phys. Rev. Lett. 71, 3202 (1993).

[12] H.E. Haber and H.A. Weldon, Phys. Rev. Lett. 46, 1497 (1981); J.I. Kapusta, Phys. Rev. D 24, 426 (1981).

[13] E. Babaev, Int. J. Mod. Phys. A 16, 1175 (2001); P. Castorina, G. Nardulli, and D. Zappala, Phys. Rev. D 72, 076006 (2005).

[14] E. Braaten and R.D. Pisarski, Phys. Rev. D 45, R1827 (1992).

[15] M. Asakawa and T. Hatsuda, Phys. Rev. Lett. 92, 012001 (2004); S. Datta et al., Phys. Rev. D 69, 094507 (2004); T. Umeda, K. Nomura, and H. Matsufuru, Eur. Phys. J. C 39S1, 9 (2005).

[16] E.V. Shuryak and I. Zahed, Phys. Rev. C 70, 021901(R) (2004); Phys. Rev. D 70, 054507 (2004). A deep attractive $q q$ potential which may be relevant to bound states is observed on a lattice; A. Nakamura and T. Saito, Prog. Theor. Phys. 112, 183 (2004). 